\title{
Geometry of complexity in conformal field theory
}

\author{
Mario Flory $\oplus^{1, *}$ and Michal P. Heller $\circledast^{2, \dagger}$ \\ ${ }^{1}$ Institute of Physics, Jagiellonian University, 30-348 Kraków, Poland \\ ${ }^{2}$ Max Planck Institute for Gravitational Physics (Albert Einstein Institute), 14476 Potsdam-Golm, Germany
}

(Received 22 July 2020; accepted 1 December 2020; published 30 December 2020)

\begin{abstract}
We initiate quantitative studies of complexity in $(1+1)$-dimensional conformal field theories with a view that they provide the simplest setting to find a gravity dual to complexity. Our work pursues a geometric understanding of the complexity of conformal transformations and embeds Fubini-Study state complexity and direct counting of stress tensor insertion in the relevant circuits in a unified mathematical language. In the former case, we iteratively solve the emerging integrodifferential equation for sample optimal circuits and discuss the sectional curvature of the underlying geometry. In the latter case, we recognize that optimal circuits are governed by Euler-Arnold type equations and discuss relevant results for three well-known equations of this type in the context of complexity.
\end{abstract}

DOI: 10.1103/PhysRevResearch.2.043438

\section{INTRODUCTION}

One of the most perplexing recent results in quantum gravity are holographic complexity proposals. They are convincing albeit speculative relations between volumes [1-3] or actions [4,5] in anti-de Sitter space-times [6-8] and hardness of preparing states in dual quantum field theories (QFTs).

Holographic complexity proposals not only call for a better understanding and explicit derivation, but also motivate searching for other manifestations of complexity in gravity. However, advancing the broadly defined field of holographic complexity requires expanding our knowledge of complexity in QFT. The aim of the present work is to initiate quantitative studies of physical notions of complexity in what arguably is the most universal setting of all conformal field theories in $1+1$ space-time dimensions $\left(\mathrm{CFTs}_{1+1}\right)$ and local conformal transformations generated by the energy-momentum tensor.

Complexity in its native quantum computing setting concerns hardness of approximating a given unitary using circuits composed from discrete gates acting only on a limited number of qubits (circuit complexity) or approximating a desired quantum state using such circuits acting on a simple state (state complexity).

Motivated by holographic complexity proposals $[9,10]$ initiated studies of complexity in QFTs. These works view the preparation of a unitary operator $U$ or, upon acting on a

*mflory@th.if.uj.edu.pl

†michal.p.heller@aei.mpg.de; On leave from: National Centre for Nuclear Research, 02-093 Warsaw, Poland.

Published by the American Physical Society under the terms of the Creative Commons Attribution 4.0 International license. Further distribution of this work must maintain attribution to the author(s) and the published article's title, journal citation, and DOI. Open access publication funded by the Max Planck Society. reference state $|\mathcal{R}\rangle$, also (target) state preparation $|\mathcal{T}\rangle=$ $U|\mathcal{R}\rangle$, in a continuous way as a path-ordered exponential

$$
U(\tau)=\overleftarrow{\mathcal{P}} e^{-i \int_{0}^{\tau} Q(\gamma) d \gamma}
$$

with $U(\tau=1)$ being equal to some desired unitary $U$. In this equation, the Hermitian operator $Q(\tau) d \tau$ is a single layer of the circuit parametrized by the parameter $\tau$ that constructs $U$. The idea used in Refs. $[9,10]$ to define complexity in a QFT appeared earlier in Refs. [11-13] as a way of bounding complexity of discrete circuits acting on qubits. The relevant definition assigns a cost to $Q(\tau)$ which reflects the decomposition of $Q(\tau)$ into more elementary building blocks (gates), each with a specified cost, and minimizes the sum of the contributions from all layers of the circuit subject to appropriate boundary conditions. Most of the studies to date were concerned with free QFTs and claimed optimality of circuits with $Q(\tau)$ being at most quadratic in underlying bosonic or fermionic operators. Such studies, despite their simplicity, could be fine-tuned to reproduce predictions of holographic complexity proposals $[9,10,14-18]$.

In our eyes, studies of complexity in QFT are similar to the development of entanglement entropy in the same context. The latter also arose in connection with black hole physics [19-21] and later became an independent subject with a strong quantum gravity component. One of the most fruitful seeds of progress in this discipline originated from the studies of entanglement entropy in the setting of CFTs ${ }_{1+1}$. This includes in particular the universal result for single interval entanglement entropy in the vacuum [21,22]. Drawing a parallel from the field of entanglement entropy, which includes also the matching of the results of $[21,22]$ by the holographic entanglement entropy [23], CFTs 1 $_{1+1}$ should indeed be viewed as an ideal setting for accelerating our understanding of complexity in QFTs and holography.

This vision is shared by Refs. [24-29], and the challenges encountered in these works directly motivate our approach. 
These include badly posed variational problems, assigning cost predominantly to trivial $U(1)$ factors in (1), or finding optimal circuits only to leading order for infinitesimal transformations. In the present work, we overcome these challenges and present a unified, geometric and quantitative view on complexity in $\mathrm{CFTs}_{1+1}$.

The universality of $\mathrm{CFTs}_{1+1}$ stems from their stress-energy tensor generating the Virasoro algebra. Adopting the setting of Refs. [25,29], we will be concerned with unitary circuits obtained from the exponentiation of the stress-energy tensor but employ a different way of assigning a cost to the involved operations. We focus on a $\mathrm{CFT}_{1+1}$ defined on a Lorentzian cylinder, whose circle has a unit radius and is parametrized by the coordinate $\sigma$. We will also restrict ourselves to one copy of the Virasoro algebra. This means we will assign complexities to unitary circuits of the form (1) on a representation of the group of diffeomorphisms on the circle, where an operator $U(\tau)$ corresponds to a group element $f(\tau, \sigma)$ that maps the circle to itself. The function $f(\tau, \sigma)$ hence represents a sequence of diffeomorphisms of $\sigma$ interpolating between the identity $f(0, \sigma)=\sigma$ and a desired one $f(1, \sigma) \equiv f(\sigma)$. Note that we will ignore terms stemming from the central extension of the group, as these would lead to additional complex phase factors in (1), which are generally considered to be irrelevant for physical notions of complexity (a problem faced in Refs. [25,29]). An infinitesimal layer of the circuit is generated by

$$
Q(\tau)=\int_{0}^{2 \pi} \frac{d \sigma}{2 \pi} T(\sigma) \epsilon(\tau, \sigma),
$$

where $T(\sigma)$ is the right-moving component of the stressenergy tensor operator and $\epsilon(\tau, \sigma)$ is an element of the Lie-algebra defined via

$$
\epsilon(\tau, f(\tau, \sigma))=\dot{f}(\tau, \sigma) .
$$

In this paper, we discuss two viable instances of cost functions. The first emerges by taking an energy eigenstate $|h\rangle$ and evaluating the Hilbert space distance traversed by the circuit defined by (1) and (2). This is the Fubini-Study complexity defined in Ref. [9]. The second instance realizes the approach of Ref. [10] and arises from treating $T(\sigma)$ as a one-parameter set of elementary contributions to each circuit layer and minimizing the $L_{2}$ norms of $\epsilon(\tau, \sigma), \epsilon^{\prime}(\tau, \sigma)$, or a combination of both, averaged over all circuit layers.

Our approach is the first study of complexity in a generic (including large central charge $c$ ) $\mathrm{CFT}_{1+1}$ that (1) does not assign cost to trivial factors (see Ref. [29]), (2) has a well-posed variational problem for determining a transformation between two arbitrary unitaries generated by the insertions of $T(\sigma),(3)$ sheds light on the underlying geometry of circuits by probing its sectional curvatures, and (4) selects the physically desirable notions of complexity based on the properties of optimal circuits. While we intend to present our main results in a concise and self-contained manner, some further discussions can be found in our companion work [30].

\section{COST FUNCTIONS AND COMPLEXITY}

The Fubini-Study metric also known as fidelity susceptibility arises from considering an overlap between two nearby states in the Hilbert space [31]. It is attractive from the point of view of holography and the largely open problem of physical interpretation of holographic complexity proposals, since it is known how the overlap between at least certain states in holographic QFTs manifest itself on the gravity side [26]. For a family of states $|\psi(\tau)\rangle$ parametrized by $\tau$, we can define

$$
|\langle\psi(\tau) \mid \psi(\tau+d \tau)\rangle| \approx 1-G_{\tau \tau}(\tau) d \tau^{2}+\mathcal{O}\left(d \tau^{3}\right),
$$

where $G_{\tau \tau} \geqslant 0$ is the Fubini-Study metric. Assume $|\psi(\tau)\rangle$ is a path on the space of states parametrized by unitary operators acting on an initial state $|h\rangle$,

$$
|\psi(\tau)\rangle \equiv U(\tau)|h\rangle .
$$

The Fubini-Study metric $G_{\tau \tau}$ becomes then the variance of $Q(\tau)$ evaluated in the state $|\psi(\tau)\rangle[25]$ or, introducing

$$
\tilde{Q}(\tau)=U(\tau)^{\dagger} Q(\tau) U(\tau),
$$

equivalently the variance of $\tilde{Q}(\tau)$ evaluated in the state $|h\rangle$ : $\left\langle h\left|\tilde{Q}^{2}(\tau)\right| h\right\rangle=\left\langle\psi(\tau)\left|Q^{2}(\tau)\right| \psi(\tau)\right\rangle$ by definition, and similarly for the one-point function. Note that the applications of the operators $U(\tau)$ in (6), using (2), essentially causes a conformal transformation of the stress-energy tensor. Using the well-known transformation law and ignoring the Schwarzian term leading to an irrelevant for us phase factor, we can write [25]

$$
\tilde{Q}(\tau)=\int_{0}^{2 \pi} \frac{d \sigma}{2 \pi} T(\sigma) \frac{\dot{f}(\tau, \sigma)}{f^{\prime}(\tau, \sigma)} .
$$

Each trajectory through state space $|\psi(\tau)\rangle$ parametrized by $\tau \in[0,1]$ can now be assigned the total cost $\mathrm{L}_{\mathrm{FS}}$

$$
\mathrm{L}_{\mathrm{FS}}=\int_{0}^{1} d \tau \sqrt{G_{\tau \tau}(\tau)}
$$

and complexity arises as its minimum subject to the appropriate boundary conditions [9]. We should note here that the present discussion is completely general and concerns complexity of state $|\mathcal{T}\rangle=U|h\rangle$ given a reference state $|\mathcal{R}\rangle=|h\rangle$. Alternatively, one can view it as a definition of circuit complexity associated with a circuit representation of a unitary $U$ in which one decomposes $\tilde{Q}(\tau)$ into elementary transformations. This is similar to the notion of circuit complexity explored in Ref. [28].

One can alternatively define an a priori inequivalent notion of complexity based on a variance of $Q(\tau)$ in the state $|h\rangle$ [instead of $|\psi(\tau)\rangle$ as so far], which would be a more faithful realization of the approach [10]. What we mean by that is that the cost of one layer in the Fubini-Study metric depends not only on $\epsilon(\tau, \sigma)$ from a given layer, but also on what all previous layers do through the two-point function of $T$ in the evolved state $|\psi(\tau)\rangle$. On the other hand, in the approach of Ref. [10] the cost of each layer depends only on $\epsilon(\tau, \sigma)$ as the insertions of $T$ would be assigned the same weight at each layer.

The discussion so far was completely general, but now we specialize to circuits defined by (7). Our choice for $|h\rangle$, as in Refs. [25,29], is that of an energy eigenstate in the $\mathrm{CFT}_{1+1}$ corresponding, via the operator-state correspondence, to a (quasi)primary of the chiral dimension $h$. To evaluate (8) explicitly, we follow Refs. [25,29] and write the variance of $\tilde{Q}$ 
as a bilocal integral

$$
\mathrm{L}=\int_{0}^{1} \frac{d \tau}{2 \pi} \sqrt{\iint_{0}^{2 \pi} d \sigma d \kappa \frac{\dot{f}(\tau, \sigma)}{f^{\prime}(\tau, \sigma)} \frac{\dot{f}(\tau, \kappa)}{f^{\prime}(\tau, \kappa)} \Pi(\sigma-\kappa)},
$$

where $\Pi$ corresponds to a connected correlator of the stressenergy tensor in the state $|h\rangle[32]$

$$
\begin{aligned}
\Pi(\sigma-\kappa) & =\langle h|T(\sigma) T(\kappa)| h\rangle-\langle h|T(\sigma)| h\rangle\langle h|T(\kappa)| h\rangle \\
& =\frac{c}{32 \sin ^{4}[(\sigma-\kappa) / 2]}-\frac{h}{2 \sin ^{2}[(\sigma-\kappa) / 2]} .
\end{aligned}
$$

As usual when studying geodesic motion, by requiring affine parametrization we can move from a length-functional (8) to an energy-functional where, essentially, the square root in (8) and (9) is removed. To do so, we note that (9) clearly corresponds to a geodesic problem in infinite dimensions, where summation over indices has been replaced by integration over variables $\sigma, \kappa, f(\tau, \sigma)$ has taken over the role of the coordinate $X^{\sigma}(\tau)$, and the expression $\frac{\Pi(\sigma-\kappa)}{f^{\prime}(\tau, \sigma) f^{\prime}(\tau, \kappa)}$ plays the role of the metric $g_{\sigma \kappa}(X(\tau))$. The partial integrodifferential equation (IDE) of motion for the circuit $f(\tau, \sigma)$ extremizing (9) then reads

$$
\begin{gathered}
\int_{0}^{2 \pi} d \sigma\left(-\Pi(\sigma-\kappa) \frac{d}{d \tau}\left(\frac{\dot{f}(\tau, \sigma)}{f^{\prime}(\tau, \sigma) f^{\prime}(\tau, \kappa)}\right)\right. \\
\left.+\frac{\dot{f}(\tau, \sigma)}{f^{\prime}(\tau, \sigma)} \partial_{\kappa}\left(\Pi(\sigma-\kappa) \frac{\dot{f}(\tau, \kappa)}{f^{\prime}(\tau, \kappa)^{2}}\right)\right) \equiv 0 .
\end{gathered}
$$

As expected, this equation is of second order in derivatives with respect to $\tau$. This is adequate for a boundary value problem in which we envision being given an initial and final condition, $f(0, \sigma)$ and $f(1, \sigma)$, and finding the shortest circuit $f(\tau, \sigma)$ connecting these two maps. This is a notable contrast to the geometric actions studied in Refs. [25,29], which lead to equations first order in $\tau$, in which generally only one initial value $f(0, \sigma)$ needs to be provided to fix a solution.

It is the most natural for us to equate the integration kernel $\Pi$ with the connected stress-energy two-point function in the state $|h\rangle$ (10), however we have kept our discussion more generic for a reason. Broadly speaking, our goal in this paper is to define geodesic motion on the Virasoro group, and this has of course already been accomplished in the framework of Euler-Arnold-type partial differential equations (PDEs) such as the Korteweg-de Vries (KdV), Camassa-Holm (CH), or Hunter-Saxton (HS) equations [33]. They were already discussed in the context of QFT complexity in Refs. [25,29], see also Ref. [34]. In order to show how our functional (9) and equation (11) fit into this framework, we note that according to (9), the distance between the identity map $f=\sigma$ and a map $f=f_{1}(\sigma)$ is identical to the distance between $f=\sigma$ and the inverse map $F_{1}(\sigma)=f_{1}^{-1}(\sigma)$. This is easy to show by using invariance under a change of affine parameter $\tau \rightarrow$ $s=1-\tau$ and invariance under conformal transformations $f(\tau, \sigma) \rightarrow G(f(\tau, \sigma))$. Hence, replacing the circuit $f(\tau, \sigma)$ by the inverse circuit $F(\tau, \sigma)$ in (9) and using the identity [25]

$$
\epsilon(\tau, \sigma)=-\frac{\dot{F}(\tau, \sigma)}{F^{\prime}(\tau, \sigma)},
$$

we can write the inner product in (9) entirely in terms of the kernel $\Pi$ and the Lie-algebra-element $\epsilon$. Note that if $A=B \cdot C$, then $A^{-1}=C^{-1} \cdot B^{-1}$, hence replacing $f(\tau, \sigma)$ by $F(\tau, \sigma)$ in (9) corresponds to switching from a left- to a right-invariant metric on the Lie-group. This would yield the alternative complexity definition based on the variance of $Q$ (not $\tilde{Q}$ ) in the state $|h\rangle$ discussed below (8).

Now, while our derivation above would suggest $\Pi$ to be the stress-energy two-point function in the state $|h\rangle$ [32], another choice of

$$
\Pi(\sigma-\kappa)=a \delta(\sigma-\kappa)+b \delta^{\prime \prime}(\sigma-\kappa),
$$

with Dirac's delta function $\delta(\sigma-\kappa)$, would allow us to obtain the $\mathrm{CH}$ equation $(a=b=1)$, the HS equation $(a=0, b=1)$, and the $\mathrm{KdV}$ equation ( $a=1, b=0)$, ignoring as before the term coming from the central extension. Through the lens of Ref. [10], (13) can be seen as assigning directly a spatially uniform cost to individual insertions of $T(\sigma)$ via $Q(\tau)$ defined in (2). In a sense, our complexity functional (9) corresponds to a generalization of the inner products that led to these well studied PDEs. Likewise, while these PDEs provide valid choices for a definition of complexity for the Virasoro group, they may be also regarded as simpler models for the physics encoded in the optimization problem behind the Fubini-Study complexity defined by (11) with (10).

In the Fubini-Study case, in order to assign a well-defined finite value to (9) despite the pole of $\Pi$, we can make use of the method of differential regularization [35,36]. This means we will (implicitly) phrase the divergent terms of the twopoint function in (9) as derivatives of more mildly divergent terms and then carry the derivatives over onto the test function $\frac{\dot{f}(\tau, \sigma)}{f^{\prime}(\tau, \sigma)} \frac{\dot{f}(\tau, \kappa)}{f^{\prime}(\tau, \kappa)}$ via integration by parts. The immediate physical consequence of this is that the metric will be degenerate. For example, if $\frac{\dot{f}(\tau, \sigma)}{f^{\prime}(\tau, \sigma)}=$ const, the integrals in (9) will vanish when applying derivatives to this term due to differential regularization. Although the cause of some technical problems, this degeneracy of the metric is a desirable feature as it makes sure that transformations which only change the reference state by a complex phase will be assigned zero cost in terms of complexity. For this reason, we believe that the HS equation, $a=0$ in (13), will be a more realistic model of CFT complexity than the KdV equation studied in Refs. [25,29]. We discuss these issues in more detail in Ref. [30].

\section{OPTIMAL CIRCUITS FOR FUBINI-STUDY}

The exact solutions and properties of the $\mathrm{KdV}, \mathrm{CH}$, and HS equations are well studied [33], and hence we will now focus again on the concrete equations of motion (11) with $\Pi$ given by (10). For this, apart from trivial circuits such as $f(\tau, \sigma)=f(\tau+\sigma)$, we do not know exact solutions. However, for boundary conditions of the form $f(0, \sigma)=\sigma$, $f(1, \sigma)=\sigma+\frac{\varepsilon}{m} \sin (m \sigma)$ with $m \in \mathbb{N}, \varepsilon \ll 1$, we iteratively construct a circuit $f(\tau, \sigma)$ satisfying (11) order by order in $\varepsilon$. For example, for $m=1$, this yields

$$
\begin{aligned}
f(\tau, \sigma)= & \sigma+\varepsilon \tau \sin (\sigma) \\
& +\varepsilon^{2} \frac{c \tau^{2}-c \tau+20 h \tau^{2}-20 h \tau}{4(c+8 h)} \sin (2 \sigma)+\ldots
\end{aligned}
$$




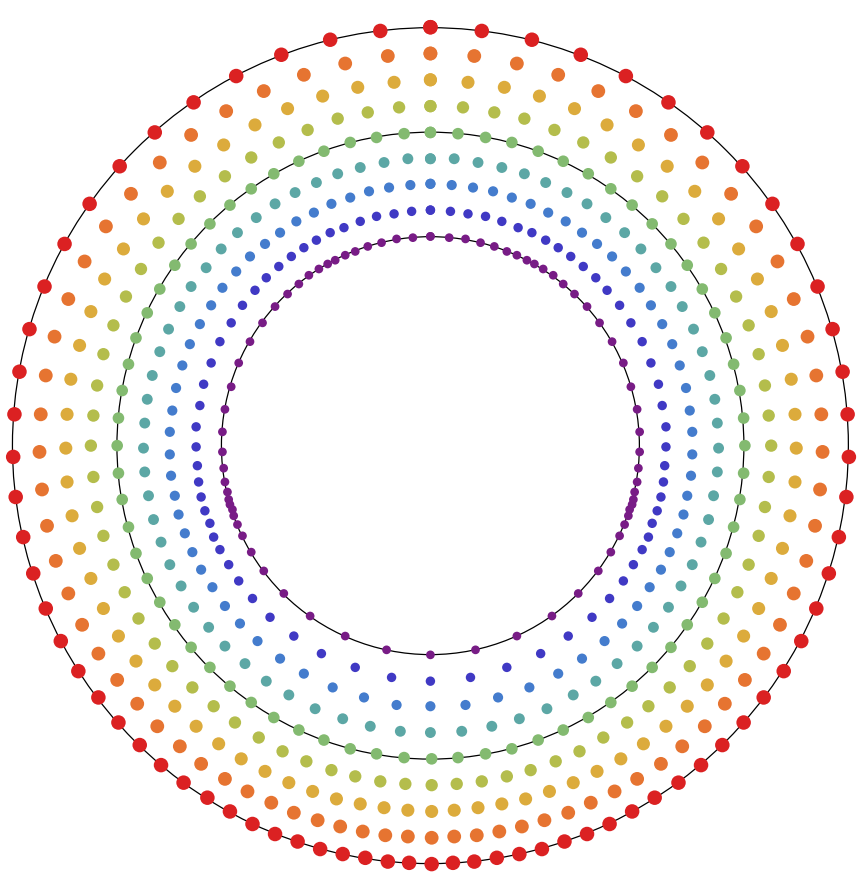

FIG. 1. A graphical representation of the circuit (14), where for simplicity we have set $c=0, h=1$ and $\epsilon=1$ in order to generate a result that is visible to the eye. For each fixed value of $\tau$, $f(\tau, \sigma)$ is a map of the circle to itself, depicted by the colored points unevenly spread out along the circles according to the function $f(\tau, \sigma)$ with evenly spaced input values for $\sigma$. The radial ordering (also emphasized by color) corresponds to the progression of the parameter $\tau$ from -1 to 1 in steps of $\Delta \tau=1 / 4$. The solid circles mark $\tau=-1,0,1$, respectively. At $\tau=0, f(\tau, \sigma)$ is the identity map by construction. "Wave breaking" happens when infinitesimally close points are mapped to the same location because $f^{\prime}(\tau, \sigma)=0$.

and the square of the on-shell Lagrangian in (9) is

$$
\begin{aligned}
& \iint d \sigma d \kappa \Pi(\sigma-\kappa) \frac{\dot{f}(\tau, \sigma)}{f^{\prime}(\tau, \sigma)} \frac{\dot{f}(\tau, \kappa)}{f^{\prime}(\tau, \kappa)} \\
& \quad=2 \pi^{2} h \varepsilon^{2}+\frac{\pi^{2}\left(3 c^{2}+56 c h+112 h^{2}\right)}{96(c+8 h)} \varepsilon^{4}+\ldots
\end{aligned}
$$

We comment more on this result and how it was obtained in the Appendix. Figure 1 shows a graphical representation of the circuit (14).

\section{GEOMETRY OF COMPLEXITY}

In order to gain a qualitative understanding of the geometry of the Fubini-Study complexity and compare with other notions, we will instead proceed to calculate the sectional curvatures $K(u, v)$ at the identity map $f(\sigma)=\sigma$ for tangent vectors of the form $u=\sin (m \sigma), v=\sin (n \sigma), m, n \in \mathbb{N}$ and $m \neq n$. Following the analogy with finite dimensional geodesic motion, first and second derivatives of the metric can then be defined as functional derivatives, e.g., $\frac{\partial g_{\sigma \kappa}}{\partial x^{\eta}} \rightarrow$ $\frac{\delta}{\delta f(\eta)} \frac{\Pi(\sigma-\kappa)}{f^{\prime}(\sigma) f^{\prime}(\kappa)}=-\Pi(\sigma-\kappa)\left(\frac{\delta^{\prime}(\sigma-\eta)}{f^{\prime}(\sigma)^{2} f^{\prime}(\kappa)}+\frac{\delta^{\prime}(\kappa-\eta)}{f^{\prime}(\sigma) f^{\prime}(\kappa)^{2}}\right)$. In order to calculate the sectional curvatures,

$$
K(u, v)=\frac{R_{\sigma \kappa \eta \omega} u^{\sigma} u^{\eta} v^{\kappa} v^{\omega}}{(u \cdot u)(v \cdot v)-(u \cdot v)^{2}},
$$

we would still need an analog of the inverse metric which is needed in the definition of the Riemann tensor $R_{\sigma \kappa \eta \omega}$. Note that in (16) the scalar product is taken using the metric $g_{\sigma \kappa}$ and we assume Einstein's summation formula involving integration. The inverse metric in question does not exist because our metric is degenerate. However, for circuits of the type (14) which satisfy the condition $\int d \sigma \frac{\dot{f}(\tau, \sigma)}{f^{\prime}(\tau, \sigma)}=0$ for all $\tau$, it is possible to show that the equation of motion (11) is left invariant when adding a nonzero constant to the two-point function $\Pi$. This creates a metric which is invertible and yet has identical (i.e., independent of the added constant) sectional curvatures to the original metric in the tangent planes spanned by $u=\sin (m \sigma), v=\sin (n \sigma)$. We find

$$
\begin{aligned}
K(u, v)= & \frac{3}{\pi^{2}(m+n)}\left[\frac{(2 m+n)(m+2 n)}{24 h+c(m+n-1)(m+n+1)}\right. \\
& \left.-\frac{(2 m-n)(m+n)^{2}}{m\left(24 h+c\left(m^{2}-1\right)\right)}\right] \text { for } m>n .
\end{aligned}
$$

For $0 \leqslant h / c<\frac{4}{13}$, all $K(u, v)$ in (17) are negative, for $h / c \rightarrow$ $+\infty$, all $K(u, v)$ are positive, and for generic $h>0, c>0$, only a finite number of $K(u, v)$ for small $m, n$ will be positive, while the infinitely many remaining ones will be negative. Due to a theorem from Ref. [37], we know that there should always be some tangent planes in which $K>0$, but the result above suggests that the generic curvature felt by the geodesic curves we are investigating will be negative unless $h / c \rightarrow$ $\infty$. This is important, as in the study of Euler-Arnold-type geodesic equations negative sectional curvatures are related to a strong dependence of geodesics on initial conditions [38]. For models of complexity, the necessity of negative sectional curvatures has been discussed in Refs. [39,40]. Curiously, the metrics underlying the $\mathrm{KdV}$ and $\mathrm{CH}$ equations lead to sectional curvatures of nondefinite sign [41,42], while the geometry underlying the HS equation is positively curved [43]. Furthermore, it was shown in Ref. [43] that the geometry underlying the HS equation maps the group manifold to an open subset of an $L^{2}$-sphere, implying geodesic incompleteness: Geodesics can leave the space of invertible maps on the circle in finite affine parameter $\tau$. From the point of view of the HS equation as a wave equation, this is related to the phenomenon of wave breaking, but from a complexity perspective this phenomenon is harder to interpret. It would be fascinating to develop a better understanding of the conditions on a generic $\Pi$ under which equations of the form (11) allow for such wave breaking in finite time $\tau$. We conjecture that this should not be the case for physically sensible choices of complexity.

\section{SUMMARY AND OUTLOOK}

We addressed the problem of complexity of unitary operators resulting from exponentiation of the right- (or left-) moving component of the stress-energy tensor in $\mathrm{CFT}_{1+1}$, see (1) and (2). There are three important features of the complexity notions we consider: Firstly, they lead to equations 
of motion second order in derivatives of $\tau$, which allows to search for optimal circuits connecting two elements of the Virasoro group. Secondly, they are based on only counting nontrivial (neglecting phase factors) elementary operations with non-negative weights. It would be interesting to see if there are circumstances under which the neglected phase factor can be interpreted as a geometric phase [44], see, e.g., Ref. [45]. Thirdly, the whole construction uses explicitly spatially local gates generated by the stress-energy operator and counts either linear combinations of it via the FubiniStudy approaches or directly the usage of local operators itself.

We started with the definition of complexity based on the Fubini-Study distance (4), as it seems to be easier to embed in holography and has other attractive properties. This naturally led us to the Euler-Arnold type nonlinear equation (11). Despite the intricate form of this equation of motion in an infinitely dimensional space, we were able to find approximate solutions interpolating between the identity and its perturbation containing a single Fourier mode, see (14). The leading term there reproduces the result of Refs. [24,26] for complexity change under infinitesimal conformal transformations, which is a double integral of two test functions (related to $\dot{f}$ ) integrated against the stress-energy two-point function as integration kernel. Higher-order terms in (14) and (15) are new predictions originating from the nonlinear nature of the equation of motion. We also evaluated the sectional curvatures at the identity and found that for the Fubini-Study metric in physically relevant cases it is negative in most directions, see (17).

Subsequently, we looked at another possibility of defining circuit complexity that is based on explicit counting of appearances of the stress-energy tensor. This approach can be thought of as originating from a state in which the correlation function of the stress-energy tensor is ultralocal (13), and it would be exciting to pursue this analogy further, perhaps along the lines of Ref. [46]. Optimal circuits in this case would be exactly described by the $\mathrm{KdV}, \mathrm{CH}$ or $\mathrm{HS}$ equations, which were suggested as models for complexity in Refs. [25,29]. However, the geometries underlying these equations may have undesirable properties from a complexity perspective, such as positive sectional curvatures or geodesic incompleteness [43].

One intriguing open problem is realizing circuits given by (1) and (2) in holography, and their relation to holographic complexity proposals [1-5]. A strong hint in this direction is the agreement noted in Ref. [26] for infinitesimal conformal transformations using the results from Ref. [24].

Another way forward is to understand the circuit (1) with $Q(\tau)$ given by (2) as being realized by a $\mathrm{CFT}_{1+1}$ in a curved geometry with $\tau$ as the physical time. This brings a parallel with the path-integral optimization program $[28,47,48]$, which was, however, predominantly presented in the context of nonunitary circuits originating from the Euclidean time evolution. Another interesting issue is the question of permissible cost functions being covariant functionals of the underlying metric [28]. Finally, it is clearly important to see if inclusion of primary operators and their descendants in circuits containing the stress-energy tensor can lead to short cuts, see Ref. [34].

\section{ACKNOWLEDGMENTS}

We would like to thank Volker Schomerus for being involved in the initial part of this collaboration. In the context of this work and its companion paper [30], we particularly benefited from correspondence with Diptarka Das and discussions with Paweł Caputa on [25]. Furthermore, we gratefully acknowledge Martin Ammon, Johanna Erdmenger, Marius Gerbershagen, Romuald Janik, and Anna-Lena Weigel for conversations and correspondence, as well as Alexandre Belin, Minyong Guo, Ro Jefferson, Javier Magán, Ali Naseh, Onkar Parrikar, Blagoje Oblak, Gábor Sárosi, and Claire Zukowski for very useful comments on both papers. The Gravity, Quantum Fields and Information (GFQI) group at AEI is supported by the Alexander von Humboldt Foundation and the Federal Ministry for Education and Research through the Sofja Kovalevskaja Award. MF was supported by the Polish National Science Centre (NCN) grant 2017/24/C/ST2/00469. M.F. is also grateful to the organizers of the GQFI Workshop IV, where this work was presented for the first time, and the GFQI group at AEI for its hospitality.

\section{APPENDIX}

In this extra section, we will further elaborate on the iterative procedure which led us to the approximate solution (14), see also Ref. [30] for further details.

Suppose we want to calculate the geodesic circuit from $f_{0}(\sigma)=\sigma$ at $\tau=0$ to some target map of our choice, written as

$$
f_{1}(\sigma)=\sigma+\varepsilon g(\sigma)
$$

at $\tau=1$ with $\varepsilon \ll 1$. To lowest order in $\varepsilon$, the geodesic circuit connecting these two maps will just be the linear interpolation between them, and for higher-order corrections in $\varepsilon$ we write

$$
f(\tau, \sigma)=\sigma+\tau \varepsilon g(\sigma)+\varepsilon^{2} f^{(2)}(\tau, \sigma)+\ldots
$$

This ansatz alone stills lead to integrodifferential equations for $f^{(n)}$ at increasing orders in $\varepsilon$. In addition, we represent $f^{(n)}(\tau, \sigma)$ as a Fourier series

$$
\begin{aligned}
f^{(n)}(\tau, \sigma)= & b_{n, 0}(\tau)+\sum_{j \in \mathbb{N}} b_{n, j}(\tau) \cos (j \sigma) \\
& +\sum_{j \in \mathbb{N}} a_{n, j}(\tau) \sin (j \sigma) .
\end{aligned}
$$

Instead of one integrodifferential equation of motion for $f^{(n)}(\tau, \sigma)$, we obtain an infinite number of coupled ordinary differential equations (ODEs) for the modes $b_{n, 0}(\tau), b_{n, j}(\tau)$ and $a_{n, j}(\tau)$. The term $b_{n, 0}(\tau)$ can be set equal to zero at every order without loss of generality for reasons explained in Ref. [30]. The other modes should be solved for subject to the conditions

$$
a_{n, j}(0)=a_{n, j}(1)=b_{n, j}(0)=b_{n, j}(1)=0 .
$$

For the moment, we make the additional assumption that $g(\sigma)$ in (A1) is proportional to one single Fourier mode: $g(\sigma)=\sin (m \sigma)$ with some integer $m$. The benefit of this assumption is that the on-shell (squared) Lagrangian under 
the ansatz (A2) can be calculated explicitly order by order in $\varepsilon$, and equations of motion can then be calculated for the modes $a_{n, j}(\tau), b_{n, j}(\tau)$ by varying this expression for the Lagrangian. For the form of $g(\sigma)$, which we have chosen, and in fact for any $g(\sigma)$ which has a Fourier series that exactly terminates after a finite mode number $m$, it can be shown that there will be an integer number $M(m, n)$ such that the modes $a_{n, j}(\tau), b_{n, j}(\tau)$ decouple from all other modes for $j \geqslant$ $M(m, n)$, obtaining trivial equations $\ddot{a}_{n, j}=0=\ddot{b}_{n, j}$, which, subject to (A4), simply imply $a_{n, j}=0=b_{n, j}$.

Hence, at any finite order $n$ of $\varepsilon$, we only have to solve a finite system of coupled ordinary differential equations for the $a_{n, j}(\tau), b_{n, j}(\tau)$ with $j<M(m, n)$, which is tedious but straightforwardly doable. Higher and higher orders in $\varepsilon^{n}$ can then be added iteratively. For the specific choice of $g(\sigma)=$ $\sin (\sigma)(m=1)$, we obtained the solution (14) in this way.

What is now the distance between $f(0, \sigma)=\sigma$ and $f(1, \sigma)=\sigma+\varepsilon \sin (\sigma)$ ? As the Lagrangian is conserved by affine parametrization and the curve is parametrised such that it reaches its destination at $\tau=1$, the square of the FubiniStudy distance (8) is just (up to a factor $4 \pi^{2}$ ) equal to the value $\mathrm{L}_{s q}$ of the square of the Lagrangian from (9), i.e., (15). As expected, for small $\varepsilon$ the distance $\mathrm{L}_{F S} \propto \sqrt{\mathrm{L}_{s q}}$ will be linear in $\varepsilon$.

We will now list a few important observations about this result and the iterative method in general.

(1) While we assume $\varepsilon \ll 1$, in principle, we can go to arbitrarily high orders in $\varepsilon^{n}$. This means that even when the target map (A1) is not infinitesimally close to the identity, we can calculate the extremal circuit to arbitrary precision, modulo possible issues arising in the presence of conjugate points, which we leave for future study. Furthermore, as the extremal circuit is a minimizer of length, even finite accuracy approximations could be useful in providing upper bounds on the distance which become increasingly tight as more and more orders of $\varepsilon$ are added.

(2) Of course, if only an approximation to the circuit with finite precision is needed, the equations of motion might be solved numerically instead. Besides some technical problems pointed out in Ref. [30], this would require a shooting or relaxation method while our iterative method is by design well adapted to the situation where initial and final conditions are given for the circuit (instead of initial position and velocity), which is the most physical setup for a problem of this kind. Also, the iterative method produces analytical results (as series expansions in $\varepsilon$ ), which may be inherently an advantage in some circumstances.

(3) The first point above concerns the issue of what the convergence radius is for the seriesexpansion in (15). For $\varepsilon=1, f^{\prime}(1, \sigma)=1+\cos (\sigma)$ can be zero for some $\sigma=\sigma_{0}$, and such maps with $f^{\prime}\left(\sigma_{0}\right)=0$ are not proper group elements as they would not be invertible one-to-one maps of the circle to itself. A very important question is whether this boundary of the space of allowed maps can be reached in finite distance, which is indeed possible in the case of the HS equation as shown in Ref. [43]. Based on our physical intuition for complexity, we would like to conjecture that this is not the case. This would require that the series in (15) has a finite convergence radius $\varepsilon<1$ and describes an analytic function with a pole at $\varepsilon=1$. We hope to study this issue in more detail in the future.

(4) In the above, we have set $g(\sigma)=\sin (m \sigma)$ as a simple example and proof of concept. However, our iterative procedure is much more versatile. Firstly, we could straightforwardly use any function $g(\sigma)$ for which the Fourier series terminates after a finite mode number (even though in practice it adds a lot of workload).

However, even more generally, consider a target map with nonterminating Fourier series

$$
g(\sigma)=\sum_{m \in \mathbb{Z}} c_{m} e^{i m \sigma} .
$$

For this to converge to a smooth function $g \in C^{\infty}$, we need $\sum_{m}\left|c_{m}\right||m|^{N}<\infty$ for any integer $N$. For concreteness, we can consider the case $c_{m}=\hat{c}_{m} q^{m}$ with some $0<q<1$ and $\hat{c}_{m}=\mathcal{O}(1)$ for any $m$. In this case, we can simply replace $q$ with $\varepsilon$, and do the iterative procedure as before, imposing adequate boundary conditions on all modes at any given order. Essentially, as $\lim _{m \rightarrow \infty} c_{m}=0$, the Fourier series (A5) can be interpreted as always effectively terminating for any given finite level of accuracy (determined by the magnitude of $\varepsilon^{n}$ ).

(5) Of course, in our eyes there is no "best" or "right" choice of $g(\sigma)$. The most important possible achievement would be a comparison with bulk results, for example along the lines of the comparison between the leading order results of Refs. [24,26], but for higher orders in $\varepsilon$. For this task a simple function like $g(\sigma)=\sin (m \sigma)$ would be sufficient for obtaining nontrivial results, and we see no reason why a more complicated function $g(\sigma)$ would give any additional benefits beyond that.

(6) In (15). we worked with the square of the original Lagrangian, which is also more convenient for deriving the equations of motion. However, we have so far left ambiguous whether the actual value of the complexity should be calculated from this squared Lagrangian as in (15), or the original Lagrangian including the square root (9). The reason for this is that assuming affine parametrization, the equations of motion following from both kinds of Lagrangian are equivalent, and so are hence their solutions and the sensitivity of the geodesic problem to initial conditions [related to the sectional curvatures (17)], which was a focus of our paper. It is our philosophy that the study of such geometric properties of a given complexity proposal is as important as the motivation of the employed cost function itself, in contrast to some parts of the holographic literature that seem to end their investigations at the point where they have motivated a choice of cost function, without analyzing the equations of motion, their generic solutions and underlying geometry in detail.

See Ref. [49] for a discussion of the issue of distance functionals (i.e., Lagrangians including a square root) versus energy functionals (i.e., squared Lagrangians). In our case, if we have in mind a comparison with bulk results for the complexity $=$ volume proposal along the lines of Refs. [24,26], the squared Lagrangian seems more relevant as then complexity scales linearly with the central charge instead of its square-root. In fact, our leading order result in $\varepsilon$ then trivially reproduces the results for complexity change of the ground state $(h=0)$ calculated in Refs. [24,26] for the 
complexity $=$ volume proposal and conformal transformation in only one copy of the Virasoro group (which we have exclusively considered in this paper). Extending our methodology to two copies of the Virasoro group in the most trivial way $\mathcal{C}_{\text {total }} \equiv \mathcal{C}_{\mathrm{Vir}_{1}}+\mathcal{C}_{\mathrm{Vir}_{2}}$ would unfortunately fail to reproduce the general results of Refs. [24,26] already at leading order. However, we leave it to future study whether for transformations in only one copy of the Virasoro group the volume change matches with our results beyond leading order.
[1] L. Susskind, Computational complexity and black hole horizons, Fortsch. Phys. 64, 24 (2016) [Addendum: Fortsch. Phys. 64, 44 (2016)].

[2] D. Stanford and L. Susskind, Complexity and shock wave geometries, Phys. Rev. D 90, 126007 (2014).

[3] J. Couch, W. Fischler, and P. H. Nguyen, Noether charge, black hole volume, and complexity, J. High Energy Phys. 03 (2017) 119.

[4] A. R. Brown, D. A. Roberts, L. Susskind, B. Swingle, and Y. Zhao, Holographic Complexity Equals Bulk Action? Phys. Rev. Lett. 116, 191301 (2016).

[5] A. R. Brown, D. A. Roberts, L. Susskind, B. Swingle, and Y. Zhao, Complexity, action, and black holes, Phys. Rev. D 93, 086006 (2016).

[6] J. M. Maldacena, The Large N limit of superconformal field theories and supergravity, Int. J. Theor. Phys. 38, 1113 (1999) [Adv. Theor. Math. Phys. 2, 231 (1998)].

[7] E. Witten, Anti-de Sitter space and holography, Adv. Theor. Math. Phys. 2, 253 (1998).

[8] S. S. Gubser, I. R. Klebanov, and A. M. Polyakov, Gauge theory correlators from noncritical string theory, Phys. Lett. B 428, 105 (1998).

[9] S. Chapman, M. P. Heller, H. Marrochio, and F. Pastawski, Toward a Definition of Complexity for Quantum Field Theory States, Phys. Rev. Lett. 120, 121602 (2018).

[10] R. Jefferson and R. C. Myers, Circuit complexity in quantum field theory, J. High Energy Phys. 10 (2017) 107.

[11] M. A. Nielsen, A geometric approach to quantum circuit lower bounds, arXiv:quant-ph/0502070.

[12] M. A. Nielsen, M. R. Dowling, M. Gu, and A. M. Doherty, Quantum computation as geometry, Science 311, 1133 (2006).

[13] M. A. Nielsen and M. R. Dowling, The geometry of quantum computation, arXiv:quant-ph/0701004.

[14] S. Chapman, J. Eisert, L. Hackl, M. P. Heller, R. Jefferson, H. Marrochio, and R. C. Myers, Complexity and entanglement for thermofield double states, Sci. Post. Phys. 6, 034 (2019).

[15] S. Chapman, D. Ge, and G. Policastro, Holographic complexity for defects distinguishes action from volume, J. High Energy Phys. 05 (2019) 049.

[16] E. Caceres, S. Chapman, J. D. Couch, J. P. Hernandez, R. C. Myers, and S.-M. Ruan, Complexity of mixed states in QFT and holography, J. High Energy Phys. 03 (2020) 012.

[17] A. Bernamonti, F. Galli, J. Hernandez, R. C. Myers, S.-M. Ruan, and J. Simón, First Law of Holographic Complexity, Phys. Rev. Lett. 123, 081601 (2019).

[18] A. Bernamonti, F. Galli, J. Hernandez, R. C. Myers, S.-M. Ruan, and J. Simón, Aspects of the first law of complexity, J. Phys. A: Math. Theore. 53, 294002 (2020).

[19] L. Bombelli, R. K. Koul, J. Lee, and R. D. Sorkin, A quantum source of entropy for black holes, Phys. Rev. D 34, 373 (1986).

[20] M. Srednicki, Entropy and Area, Phys. Rev. Lett. 71, 666 (1993).
[21] C. Holzhey, F. Larsen, and F. Wilczek, Geometric and renormalized entropy in conformal field theory, Nucl. Phys. B 424, 443 (1994).

[22] P. Calabrese and J. L. Cardy, Entanglement entropy and quantum field theory, J. Stat. Mech. (2004) P06002.

[23] S. Ryu and T. Takayanagi, Holographic Derivation of Entanglement Entropy from AdS/CFT, Phys. Rev. Lett. 96, 181602 (2006).

[24] M. Flory and N. Miekley, Complexity change under conformal transformations in $\mathrm{AdS}_{3} / \mathrm{CFT}_{2}$, J. High Energy Phys. 05 (2019) 003.

[25] P. Caputa and J. M. Magan, Quantum Computation as Gravity, Phys. Rev. Lett. 122, 231302 (2019).

[26] A. Belin, A. Lewkowycz, and G. Sárosi, Complexity and the bulk volume, a new York time story, J. High Energy Phys. 03 (2019) 044

[27] M. Flory, WdW-patches in $\mathrm{AdS}_{3}$ and complexity change under conformal transformations II, J. High Energy Phys. 05 (2019) 086.

[28] H. A. Camargo, M. P. Heller, R. Jefferson, and J. Knaute, Path Integral Optimization as Circuit Complexity, Phys. Rev. Lett. 123, 011601 (2019)

[29] J. Erdmenger, M. Gerbershagen, and A.-L. Weigel, Complexity measures from geometric actions on Virasoro and Kac-Moody orbits, J. High Energy Phys. 11 (2020) 003.

[30] M. Flory and M. P. Heller, Conformal field theory complexity from Euler-Arnold equations, arXiv:2007.11555.

[31] I. Bengtsson and K. Zyczkowski, Geometry of Quantum States. An Introduction to Quantum Entanglement, 2nd ed. (Cambridge University Press, Cambridge, 2017).

[32] S. Datta, P. Kraus, and B. Michel, Typicality and thermality in 2d CFT, J. High Energy Phys. 07 (2019) 143.

[33] B. Khesin and R. Wendt, The Geometry of Infinite-Dimensional Groups, A Series of Modern Surveys in Mathematics (Springer, Berlin, 2008)

[34] V. Balasubramanian, M. Decross, A. Kar, and O. Parrikar, Quantum complexity of time evolution with chaotic hamiltonians, J. High Energy Phys. 01 (2020) 134.

[35] D. Z. Freedman, K. Johnson, and J. Latorre, Differential regularization and renormalization: a new method of calculation in quantum field theory, Nucl. Phys. B 371, 353 (1992).

[36] J. I. Latorre, C. Manuel, and X. Vilasis-Cardona, Systematic differential renormalization to all orders, Annals Phys. 231, 149 (1994).

[37] J. Milnor, Curvatures of left invariant metrics on lie groups, Adv. Math. 21, 293 (1976).

[38] V. I. Arnold, Exponential Scattering of Trajectories and its Hydrodynamical Applications (Springer, Berlin, Heidelberg, 2014), pp. 419-427.

[39] A. R. Brown, L. Susskind, and Y. Zhao, Quantum complexity and negative curvature, Phys. Rev. D 95, 045010 (2017). 
[40] A. R. Brown and L. Susskind, Second law of quantum complexity, Phys. Rev. D 97, 086015 (2018).

[41] G. Misiołek, Conjugate points in the bott-virasoro group and the $\mathrm{kdv}$ equation, Proc. Am. Math. Soc. 125, 935 (1997).

[42] G. Misiolek, A shallow water equation as a geodesic flow on the bott-virasoro group, J. Geom. Phys. 24, 203 (1998).

[43] J. Lenells, The hunter-saxton equation describes the geodesic flow on a sphere, J. Geometry Phys. 57, 2049 (2007).

[44] M. V. Berry, Quantal phase factors accompanying adiabatic changes, Proc. Roy. Soc. Lond. A 392, 45 (1984).

[45] I. Akal, Reflections on Virasoro circuit complexity and Berry phase, arXiv:1908.08514.
[46] M. Miyaji, T. Numasawa, N. Shiba, T. Takayanagi, and K. Watanabe, Continuous Multiscale Entanglement Renormalization Ansatz as Holographic Surface-State Correspondence, Phys. Rev. Lett. 115, 171602 (2015).

[47] P. Caputa, N. Kundu, M. Miyaji, T. Takayanagi, and K. Watanabe, Anti-de Sitter Space from Optimization of Path Integrals in Conformal Field Theories, Phys. Rev. Lett. 119, 071602 (2017).

[48] P. Caputa, N. Kundu, M. Miyaji, T. Takayanagi, and K. Watanabe, Liouville action as path-integral complexity: From continuous tensor networks to AdS/CFT, J. High Energy Phys. 11 (2017) 097.

[49] P. Bueno, J. M. Magán, and C. Shahbazi, Complexity measures in QFT and constrained geometric actions, arXiv:1908.03577. 